\title{
Evaluation of recombinant granule antigens GRA1 and GRA7 for serodiagnosis of Toxoplasma gondii infection in dogs
}

\author{
Zedong Wang ${ }^{1,2}$, Wei Ge${ }^{2}$, Si-Yang Huang ${ }^{1}$, Jiping Li' ${ }^{2}$ Xing-Quan Zhu ${ }^{1 *}$ and Quan Liu ${ }^{2 *}$
}

\begin{abstract}
Background: Toxoplasmosis, caused by the obligate intracellular parasite Toxoplasma gondii, is an important zoonotic disease worldwide. The precise detection of T. gondii infection in dogs has important public health significance. In this study, recombinant granule antigen proteins GRA1 and GRA7 were evaluated as potential diagnostic markers for T. gondii infection in dogs by an indirect enzyme-linked immunosorbent assay (ELISA).

Results: GRA1 and GRA7 were cloned and expressed in Escherichia coli, and the recombinant GRA1, GRA7- and Toxoplasma lysate antigen (TLA)-based ELISAs were developed and evaluated using the canine positive and negative serum samples for anti-T. gondii antibodies determined by modified agglutination test (MAT) and indirect fluorescent antibody test (IFAT), showing a seroprevalence of $15.1 \%$ by TLA- and GRA1-ELISA, and 15.8\% by GRA7-ELISA, and no significant difference was observed $(P>0.05)$. When compared with the two reference assays, MAT and IFAT, the GRA7-ELISA showed the highest co-positivity and co-negativity rates. Receiver operating characteristic (ROC) analysis revealed a largest area under curve (AUC) of 0.973 ( $95 \% \mathrm{Cl}, 0.955$ to 0.991 ), and a highest relative sensitivity (93.2\%) and specificity (94.0\%) for a cut-off value of 0.809 in GRA7-ELISA.
\end{abstract}

Conclusions: The results of the present study showed that GRA7-ELISA is highly sensitive and specific, and GRA7 is a potential serodiagnostic marker for the detection of T. gondii infection in dogs.

Keywords: Toxoplasma gondii, GRA1, GRA7, Serodiagnostic marker, ELISA, Dogs

\section{Background}

Toxoplasma gondii, an obligate intracellular parasite, can infect virtually all warm-blooded animals, including humans. Humans and animals become infected by eating undercooked or raw meat containing cysts, or by consuming food contaminated with sporulated oocysts [1]. Understanding the prevalence of $T$. gondii infection in dogs is of economic and public health importance. Firstly, the infection is serious in young dogs, especially in those co-infected with canine distemper virus [2]. Secondly, dogs are potentially involved in the mechanical transmission of $T$. gondii oocysts to humans, and

\footnotetext{
*Correspondence: xingquanzhu1@hotmail.com; liuquan1973@hotmail.com 'State Key Laboratory of Veterinary Etiological Biology, Key Laboratory of Veterinary Parasitology of Gansu Province, Lanzhou Veterinary Research Institute, Chinese Academy of Agricultural Sciences, Lanzhou, Gansu Province 730046, PR China

${ }^{2}$ Military Veterinary Institute, Academy of Military Medical Sciences, Key Laboratory of Jilin Province for Zoonosis Prevention and Control, Changchun, Jilin Province 130122, PR China
}

shed parasite in their saliva [3-5]. Thirdly, in regions where dogs are used as food animals, $T$. gondii can be transmitted to humans by consumption of undercooked meat from infected dogs [6]. Lastly, dogs are the most common pets in the world, and also reflect the extent of T. gondii infection in the domestic environment [7].

Definitive diagnosis of $T$. gondii infection by mouse inoculation, or immunohistochemical analyses is optimal. However, these tests are time-consuming, involved in experimental animals, and may have a low sensitivity [8]. Multiple tests, such as indirect haemagglutination (IHA), modified agglutination test (MAT), latex agglutination test (LAT), indirect fluorescent antibody test (IFAT), and enzyme-linked immunosorbent assay (ELISA), are useful to demonstrate $T$. gondii infection in humans and animals. Despite the satisfactory results of serological tests, development of reliable and standard reagents remains a major constraint in serodiagnosis of $T$. gondii infection. Most conventional tests using tachyzoites 
grown in mice or in tissue culture are usually difficult to standardize, making the test results difficult to evaluate [9].

T. gondii dense granule antigen proteins (GRAs) are secretory proteins expressed by both tachyzoite and bradyzoite [10]. GRA1 is secreted into the parasitophorous vacuole (PV), which has $\mathrm{Ca}^{2+}$ binding domain, becoming a physiological important factor to invade in the host cells [11], and GR7 is secreted into the cytoplasm of bradyzoite-infected cells and within the PV and the PV membrane in tachyzoite-infected cells [12]. GRA1-based ELISA shows a sensitivity of about $60 \%$, but the specificity can reach $98 \%$ in humans $[13,14]$. GRA7 based-ELISA has presented overall specificity of 98 to $100 \%$ and sensitivity of 81 to $88 \%$ in humans and goats $[15,16]$. Moreover, GRA7-ELISA has the highest positive rate in pregnant women, compared with the rhoptry (ROP1), matrix antigens (MAG1), the major surface antigen (SAG1), and GRA8 [17]. However, there are few reports on evaluation of GRAs as potential diagnostic markers for T. gondii infection in dogs. In the present study, recombinant proteins GRA1 and GRA7 were expressed and evaluated for serodiagnosis of $T$. gondii infection in dogs by indirect ELISA.

\section{Methods}

\section{Ethics statement}

The pet dogs from which blood were collected, were handled in accordance with good animal practices required by the Animal Ethics Procedures and Guidelines of the People's Republic of China. The client owned pet dogs were admitted into pet hospitals in Lanzhou City, Gansu province, Northwest China. Consent was obtained from the owners of the pet dogs. The present study was approved by the Animal Ethics Committee of Lanzhou Veterinary Research Institute, Chinese Academy of Agricultural Sciences (Approval No. LVRIAEC2010-005).

\section{Preparation of Toxoplasma lysate antigen (TLA)}

T. gondii soluble antigens (TLA) was prepared by sonicating the purified $T$. gondii tachyzoites of GT1 strain, and diluted to a final concentration of $1 \mathrm{mg} / \mathrm{ml}$ in PBS as described elsewhere [18].

\section{Expression and purification of recombinant GRA1 and GRA7}

Based on the nucleotide sequence of GRA1 (HM067753) and GRA7 (JX045574), the PCR primers for amplification of the gene products of 570-bp GRA1 (forward: 5'-AACCATGGTGCGTGTGAGCGCTATTG-3'; reverse: 5'-GCGAATTCCCTCTCTCTCTCCTGTTAGGAAC-3') and 675-bp GRA7 (forward: 5'-ATTCCATGGCGGCC ACCGCGTCAGAT-3'; reverse: 5'-GCGAATTCCTCTT CTGTGTCTGTCTGCCTCTC-3') were designed. The italics in the primer sequences represented the EcoRI and NcoI linker sites in the expression vector pET-28a (Novagen). The PCR was performed at an annealing temperature of $58^{\circ} \mathrm{C}$ for $45 \mathrm{~s}$. The resulting gene products were cloned into the EcoR1/NcoI site of pET-28a to generate a recombinant plasmids pET28-GRA1 and pET28-GRA7, which were confirmed by restriction enzymes and sequencing, and further processed for the expression of recombinant products in Escherichia coli BL21 (DE3) according to the standard techniques.

The recombinant proteins were analyzed by sodium dodecyl sulfate-polyacrylamide gel electrophoresis (SDSPAGE) using a $12 \%$ polyacrylamide gel. The reactivity with $T$. gondii positive sera was tested by immunoblot. Blots of recombinant GRA1 and GRA7 were incubated with $T$. gondii-positive mice sera, followed by alkaline phosphatase-conjugated goat anti-mouse antibodies. The recombinant GRA1 and GRA7 were purified using a $\mathrm{Ni}$-NTA purification system (Qiagen, Germany) according to the manufacturer's protocol.

\section{Serum samples}

A total of 259 blood samples were collected from dogs between November and December 2010 in Lanzhou, China. Sera were separated by centrifugation at $1,500 \times \mathrm{g}$ for $10 \mathrm{~min}$ and stored at $-20^{\circ} \mathrm{C}$ until use. The positive and negative samples for $T$. gondii infection were determined by MAT and IFAT [19-21].

\section{ELISA}

Indirect ELISA was performed to test $T$. gondii infection in dogs as described by elsewhere [22]. Briefly, microplates were coated with TLA, GRA1, or GRA7, respectively. After washing, $100 \mu \mathrm{l}$ of canine serum diluted 1:50 was added to each well, and incubated for $3 \mathrm{~h}$ at $37^{\circ} \mathrm{C}$, then $100 \mu \mathrm{l}$ of horseradish peroxidase-conjugated sheep anti-dog IgG antibodies (Abcam, USA) diluted 1:20,000 was added. After incubation for $1 \mathrm{~h}$ at room temperature and washing, color was developed by the addition of a substrate solution containing tetramethylbenzidine chromogenic substrate TMB (Thermo Fisher Scientific, USA), and stopped by $2 \mathrm{M} \mathrm{H}_{2} \mathrm{SO}_{4}$. The optical densities (ODs) were measured at $450 \mathrm{~nm}$ in a microplate reader (BioTek, USA). ELISA results were determined for each serum in duplicate. The cut-off point of OD values of a positive samples was set to be at least two times higher than that of the negative samples at any dilution point.

\section{Data analysis}

The significance of association between the results of ELISAs was analyzed using the McNemar chi-square test. The degree of agreement between the results from the 2 tests was quantified using Kappa statistics. Sensitivity and specificity of ELISA was determined as described by elsewhere [23]. The expected performance of the ELISAs at different cut-off points was examined using the receiver operating characteristic (ROC) curves [24]. 


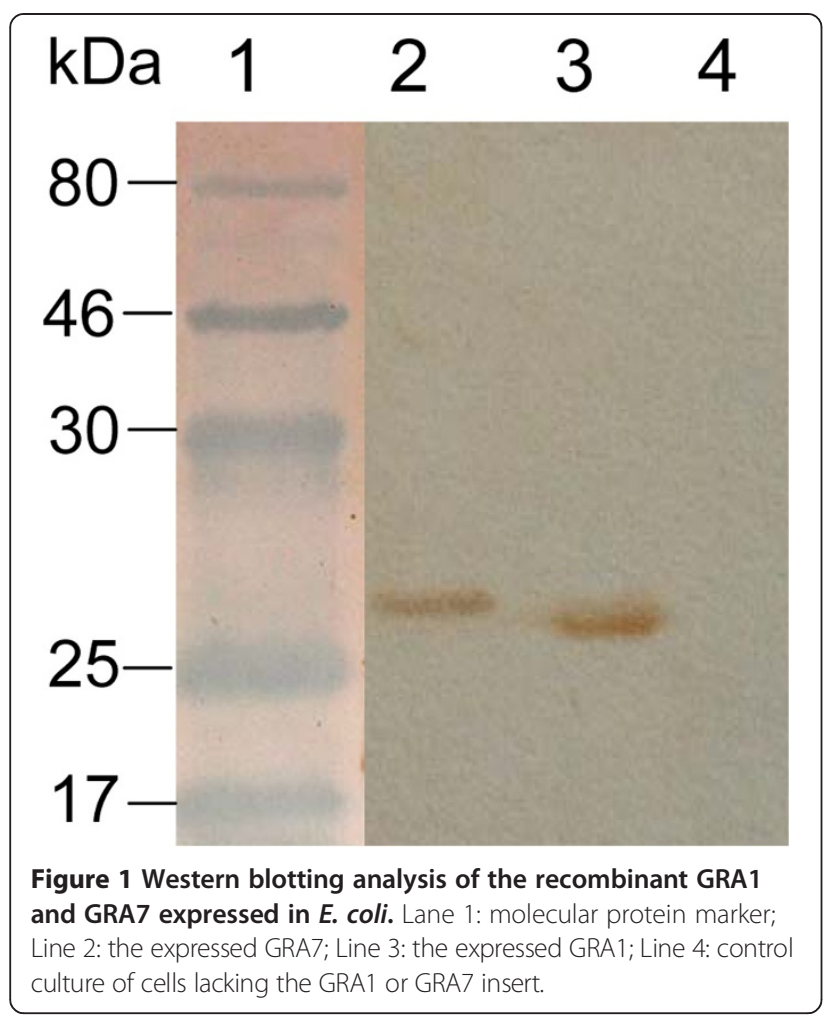

\section{Results}

Expression and purification of recombinant $T$. gondii GRA1 and GRA7

The GRA1 and GRA7 encoding genes were amplified, and cloned into expression vector pET-28a to construct recombinant plasmids pET28-GRA1 and pET28-GRA7, which were transformed into Escherichia coli BL21 (DE3), and induced by IPTG. The immunoreactivity of the

Table 1 Detection results of Toxoplamsa gondii antibodies in dogs by ELISA based on TLA, GRA1 and GRA7

\begin{tabular}{lccc}
\hline & \multicolumn{3}{c}{ MAT and IFAT } \\
\cline { 2 - 4 } & No. of positive & No. of negative & Total \\
\hline TLA-ELISA & 37 & 5 & 42 \\
No. of positive & 7 & 210 & 217 \\
No. of negative & 44 & 215 & 259 \\
Total & & & \\
GRA1-ELISA & 34 & 8 & 42 \\
No. of positive & 10 & 207 & 217 \\
No. of negative & 44 & 215 & 259 \\
Total & & & \\
GRA7-ELISA & 39 & 5 & 44 \\
No. of positive & 5 & 210 & 215 \\
No. of negative & 44 & 215 & 259 \\
Total & & &
\end{tabular}

Table 2 Prevalence of Toxoplasma gondii antibody and the titers in dogs by ELISA based on TLA, GRA1 and GRA7

\begin{tabular}{lccccc}
\hline $\begin{array}{l}\text { Detection } \\
\text { methods }\end{array}$ & $\begin{array}{c}\text { No. } \\
\text { examined }\end{array}$ & $\begin{array}{c}\text { No. (\%) } \\
\text { of positive }\end{array}$ & \multicolumn{3}{c}{$\begin{array}{c}\text { No. (\%) of samples showing } \\
\text { the antibody titers at }\end{array}$} \\
\cline { 4 - 6 } & & & $\mathbf{1 : 5 0}$ & $\mathbf{1 : 1 0 0}$ & $\mathbf{2 1 : 2 0 0}$ \\
\hline TLA-ELISA & 259 & $42(16.2)$ & $25(9.7)$ & $8(3.1)$ & $9(3.5)$ \\
GRA1-ELISA & 259 & $42(16.2)$ & $25(9.7)$ & $7(2.7)$ & $10(3.9)$ \\
GRA7-ELISA & 259 & $44(17.0)$ & $26(10.0)$ & $9(3.5)$ & $9(3.5)$ \\
\hline
\end{tabular}

expressed proteins was confirmed by Western blot analysis using the mice sera positive for anti-T. gondii IgG antibodies (Figure 1). The recombinant proteins GRA1 and GRA7 were purified using a Ni-NTA purification system, showing the purity of more than $95 \%$.

Evaluation of the diagnostic performance of TLA, GRA1 and GRA7

To evaluate the potential of recombinant proteins for serodiagnosis of $T$. gondii infection in dogs, three separate ELISAs were developed using TLA, GRA1 and GRA7 as coating antigens. The optimal working dilution, determined by checkerboard assays using serial dilutions of antigens and sera, was shown $10 \mu \mathrm{g} / \mathrm{ml}$ for TLA, and $5 \mu \mathrm{g} / \mathrm{ml}$ for both GRA1 and GRA7.

The canine serum samples were detected for anti- $T$. gondii antibodies by MAT and IFAT, showing that there were 44 positive samples and 215 negative samples. These samples were tested by TLA-, GRA1-, and GRA7ELISA, respectively. As shown in Table 1, there were 42 positive and 217 negative samples by TLA- and GRA1ELISA, while there were 44 positive and 215 negative samples tested by GRA7-ELISA (Table 1), demonstrating that the seroprevalence was $16.2 \%$ by TLA- and GRA1-ELISA, and $17.0 \%$ by GRA7-ELISA (Table 2). There was no significant difference between positive and negative results when comparing TLA-ELISA with GRA1-, and GRA7ELISA results using McNemar chi-square $(P>0.05)$. A substantial agreement (90.7\%) was found between TLAand GRA1-ELISA ( $\mathrm{kappa}=0.66 ; 95 \%$ confidence interval [CI], 0.53 to 0.79 ), and a perfect agreement (94.6\%) was observed between TLA- and GRA7-ELISA (карра = 0.80; 95\% confidence interval [CI], 0.71 to 0.90 ).

\begin{tabular}{|c|c|c|c|c|}
\hline $\begin{array}{l}\text { Detection } \\
\text { method }\end{array}$ & Sensitivity (\%) & Specificity (\%) & $\begin{array}{c}\text { False } \\
\text { positive } \\
\text { rate (\%) }\end{array}$ & $\begin{array}{c}\text { False } \\
\text { negative } \\
\text { rate (\%) }\end{array}$ \\
\hline TLA-ELISA & 88.1 & 96.8 & 11.9 & 3.2 \\
\hline GRA1-ELISA & 81.0 & 95.4 & 19.0 & 4.6 \\
\hline GRA7-ELISA & 91.0 & 97.7 & 9.0 & 2.3 \\
\hline
\end{tabular}




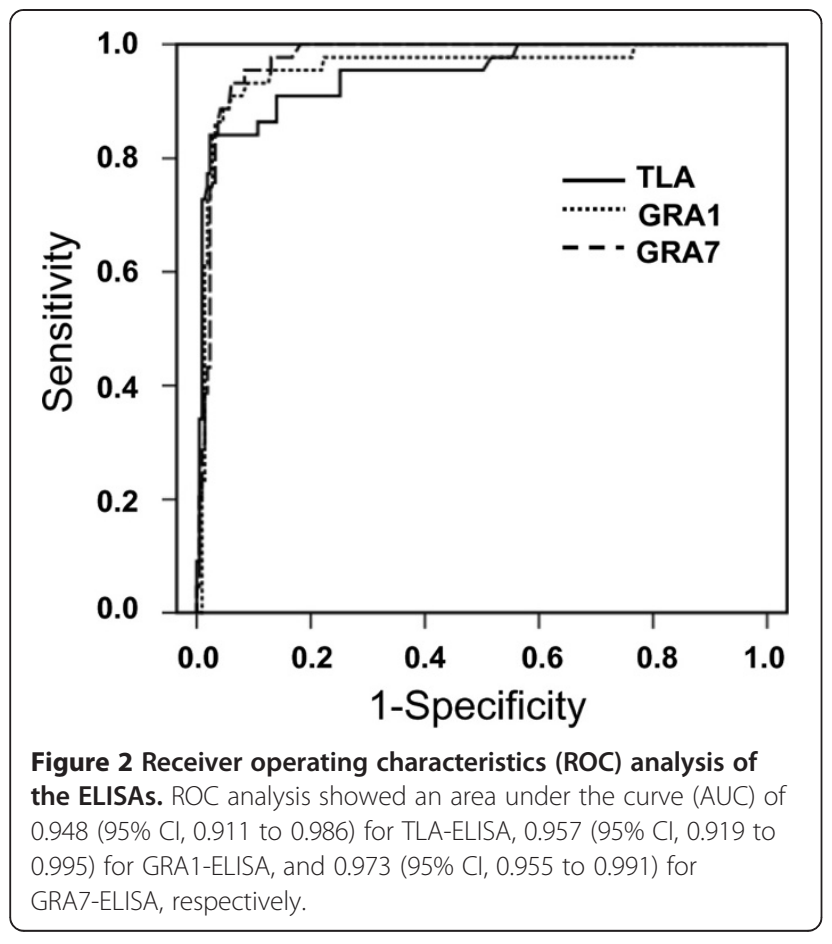

When compared with the two reference assays, MAT and IFAT, 5 false positive and 7 false negative samples were found in TLA-ELISA, 8 false positive and 10 false negative samples were found in GRA1ELISA, and 5 false positive and 5 false negative samples were found in GRA7-ELISA (Table 1), demonstrating that the GRA7-ELISA showed the highest co-positivity and co-negativity rates (Table 3 ).

\section{ROC analysis}

ROC analysis revealed an area under curve (AUC) of 0.948 (95\% CI, 0.911 to 0.986) for TLA-ELISA, 0.957 (95\% CI, 0.919 to 0.995 ) for GRA1-ELISA, and 0.973 ( $95 \%$ CI, 0.955 to 0.991 ) for GRA7-ELISA, respectively (Figure 2). The estimated sensitivities and specificities for different OD ratio cut-off values were obtained from ROC analysis (Figure 3), indicating that the cut-off value at 0.568 for TLA-ELISA shows a sensitivity of $84.1 \%$ and a specificity of $97.7 \%$, and 0.745 for GRA1-ELISA shows a sensitivity of $91.0 \%$ and a specificity of $94.0 \%$, and 0.809 for GRA7-ELISA shows a sensitivity of $93.2 \%$ and a specificity of $94.0 \%$, which were considered as the most appropriate cut-off for the 3 tests.

\section{Discussion}

Diagnosis of toxoplasmosis by demonstration of $T$. gondii is usually difficult. Bioassays using mice or cats are not suitable for rapid detection or field application, due to their price, sensitivity and the length of time to reach the results. Serological tests are still considered useful for evaluation of the infection in humans and animals. IHA, MAT, LAT, IFAT and ELISA have been used to detect $T$. gondii infection in dogs $[4,25-28]$. However, the sensitivity and specificity of these tests may vary between sampling methods, or reference populations, even when the same tests are used in different species. IHA has poor sensitivity and concordance when compared with ELISA, precluding their use for detection of T. gondii infection in dogs [29]. MAT is the most widely used methods for detection of $T$. gondii infection worldwide, as it is not species specific. However, MAT shows high false negative rate, and low agreement in dogs compared with IFAT and ELISA [20,30]. IFAT is one of the most widely used methods for detecting anti- $T$. gondii antibodies, but it is conduct only in reference laboratories, or by laboratory technicians that have enough experience for this test [31]. Thus, ELISA is recommended to detect $T$. gondii infection in dogs [30].

Most commercial ELISAs using native tachyzoite antigens may vary significantly between laboratories, or between batches. An alterative approach is to develop serological tests using recombinant proteins, with an advantage of the precise antigen composition, and easy standardization [32]. Many recombinant antigens have been produced and evaluated for serodiagnosis of $T$. gondii infection in humans and animals, including surface antigens (SAG1, and SAG2), rhoptry proteins (ROP2, and ROP4), microneme proteins (MIC1, and
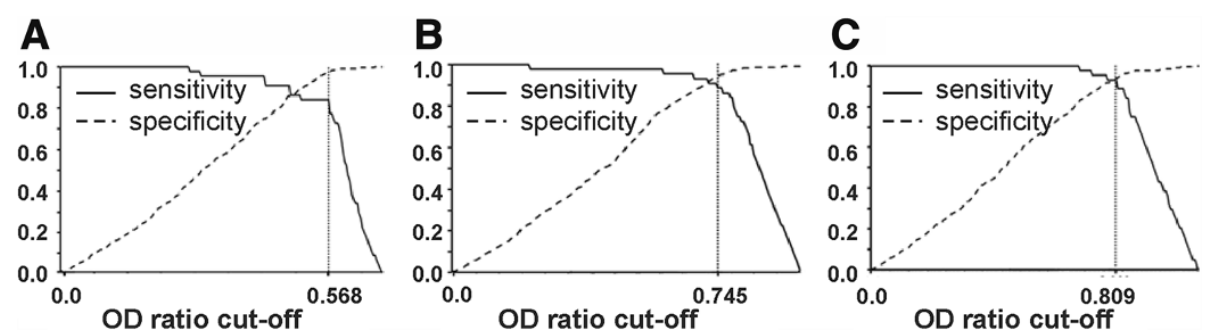

Figure 3 Relative sensitivity and specificity of TLA- GRA1 and GRA7-ELISA at different cut-offs. (A) Vertical line shows the selected cut-offs of 0.568 for TLA-ELISA. (B) Vertical line shows the selected cut-offs of 0.745 for GRA1-ELISA. (C) Vertical line shows the selected cut-offs of 0.809 for GRA7-ELISA. 
MIC3), and dense granule antigens (GRA1, GRA2, GRA5, GRA6, GRA7, and GRA8) [32,33]. Among these antigens, GRA1 is a major secretory antigen in chronic infection, and GRA7 induces a very strong antibody response in the acute infection [33]. In the present study, recombinant GRA1 and GRA7 were evaluated for serodiagnosis of $T$. gondii infection in dogs by indirect ELISA, whose results were compared with TLA-ELISA. A substantial agreement $(>90.0 \%)$ was observed among the 3 tests. If the detection results of MAT and IFAT were used as references, GRA7 showed high sensitivity and specificity as well as low FPR and FNR in comparison to GRA1 and TLA, suggesting that GRA7 is a good serological marker for the detection of $T$. gondii infection in dogs.

GRA7 is expressed by all developmental stages, including tachyzoites and bradyzoites, and is abundant on the surface and cytoplasmic matrix of host cells, the PVM, and within the PV lumen [17]. GRA7 can be used to detect anti- $T$. gondii antibodies in chronic and acute infection, but it is more associated with acute infection [34,35]. In humans infected with T. gondii, GRA7 appears significantly earlier than other antigens, such SAG1 and MAG1 [36,37]. When GRA7 is released from tachyzoites and bradyzoites, it has direct contact with the host immune system, and induces strong antibody responses in both early and late stages of infection $[12,15]$. The antigenic properties of GRA7 make it a good serological maker for the detection of anti- $T$. gondii antibodies in chronic and acute infection.

ROC analysis enabled us to compare the relative sensitivity and specificity of the developed ELSIAs at various cut-offs, in which the AUC represents a statistical summary of the overall diagnostic performance of the test $[38,39]$. In this study, the AUC of 0.973 for GRA7-ELISA suggests that it represents a highly accurate test with good discrimination of positive from negative samples, and the cut-off at 0.809 showed the most appropriate sensitivity of $93.2 \%$ and specificity of $94.0 \%$, respectively.

\section{Conclusion}

The present study, for the first time, evaluated the dense granule antigens GRA1 and GRA7 as potential diagnostic markers for T. gondii infection in dogs by ELISA, showing that the GRA7-ELISA is highly sensitive and specific, and that GRA7 is a potential serodiagnostic marker for the detection of $T$. gondii infection in dogs.

\section{Competing interests}

The authors declare that they have no competing interests.

\section{Authors' contributions}

QL and XQZ conceived and designed the study. ZW, WG and SYH performed the experiments, analyzed the data. JL helped in the study design and manuscript revision. QL and XQZ wrote the manuscript. All authors read and approved the final manuscript.

\section{Acknowledgments}

This study was supported by the Special Fund for Agro-scientific Research in the Public Interest (Grant No. 201303042), the National Natural Science Foundation of China (Grant No. 31372430), the Open Funds of State Key Laboratory of Veterinary Etiological Biology, Lanzhou Veterinary Research Institute, Chinese Academy of Agricultural Sciences (SKLVEB2013KFKT006) and the Science Fund for Creative Research Groups of Gansu Province (Grant No. 1210RJIA006).

Received: 21 March 2014 Accepted: 8 July 2014

Published: 12 July 2014

\section{References}

1. Zhou P, Chen Z, Li HL, Zheng H, He S, Lin RQ, Zhu XQ: Toxoplasma gondii infection in humans in China. Parasit Vectors 2011, 4:165.

2. Headley SA, Alfieri AA, Fritzen JT, Garcia JL, Weissenbock H, da Silva AP, Bodnar L, Okano W, Alfieri AF: Concomitant canine distemper, infectious canine hepatitis, canine parvoviral enteritis, canine infectious tracheobronchitis, and toxoplasmosis in a puppy. J Vet Diagn Invest 2013, 25:129-135.

3. Schares G, Pantchev N, Barutzki D, Heydorn AO, Bauer C, Conraths FJ: Oocysts of Neospora caninum, Hammondia heydorni, Toxoplasma gondii and Hammondia hammondi in faeces collected from dogs in Germany. Int J Parasitol 2005, 35:1525-1537.

4. Li B, Zhong N, Peng W, Shang L, Jin H, Liu Q: Seroprevalence of Toxoplasma gondii infection in dogs in Sichuan Province, southwestern China. J Parasitol 2012, 98:209-210.

5. Lindsay DS, Dubey JP, Butler JM, Blagburn BL: Mechanical transmission of Toxoplasma gondii oocysts by dogs. Vet Parasitol 1997, 73:27-33.

6. Bronson E, Emmons LH, Murray S, Dubovi EJ, Deem SL: Serosurvey of pathogens in domestic dogs on the border of Noel Kempff Mercado National Park, Bolivia. J Zoo Wildl Med 2008, 39:28-36.

7. Esch KJ, Petersen CA: Transmission and epidemiology of zoonotic protozoal diseases of companion animals. Clin Microbiol Rev 2013, 26:58-85.

8. Piergili Fioretti D: Problems and limitations of conventional and innovative methods for the diagnosis of toxoplasmosis in humans and animals. Parassitologia 2004, 46:177-181.

9. Kotresha D, Noordin R: Recombinant proteins in the diagnosis of toxoplasmosis. APMIS 2010, 118:529-542.

10. Nam HW: GRA proteins of Toxoplasma gondii: maintenance of host-parasite interactions across the parasitophorous vacuolar membrane. Korean J Parasitol 2009, 47:S29-S37.

11. Cesbron-Delauw MF, Guy B, Torpier G, Pierce RJ, Lenzen G, Cesbron JY, Charif H, Lepage P, Darcy F, Lecocq JP: Molecular characterization of a 23-kilodalton major antigen secreted by Toxoplasma gondii. Proc Natl Acad Sci U S A 1989, 86:7537-7541.

12. Jacobs D, Dubremetz JF, Loyens A, Bosman F, Saman E: Identification and heterologous expression of a new dense granule protein (GRA7) from Toxoplasma gondii. Mol Biochem Parasitol 1998, 91:237-249.

13. Lecordier L, Fourmaux MP, Mercier C, Deheca E, Masy E, Cesbron-Delauw MF: Enzyme-linked immunosorbent assays using the recombinant dense granule antigens GRA6 and GRA1 of Toxoplasma gondii for detection of immunoglobulin G antibodies. Clin Diagn Lab Immunol 2000, 7:607-611.

14. Ferrandiz J, Mercier C, Wallon M, Picot S, Cesbron-Delauw MF, Peyron F: Limited value of assays using detection of immunoglobulin $\mathrm{G}$ antibodies to the two recombinant dense granule antigens, GRA1 and GRA6 Nt of Toxoplasma gondii, for distinguishing between acute and chronic infections in pregnant women. Clin Diagn Lab Immunol 2004, 11:1016-1021.

15. Jacobs D, Vercammen M, Saman E: Evaluation of recombinant dense granule antigen 7 (GRA7) of Toxoplasma gondii for detection of immunoglobulin $\mathrm{G}$ antibodies and analysis of a major antigenic domain. Clin Diagn Lab Immunol 1999, 6:24-29.

16. Velmurugan GV, Tewari AK, Rao JR, Baidya S, Kumar MU, Mishra AK: High-level expression of SAG1 and GRA7 gene of Toxoplasma gondii (Izatnagar isolate) and their application in serodiagnosis of goat toxoplasmosis. Vet Parasitol 2008, 154:185-192.

17. Pfrepper Kl, Enders G, Gohl M, Krczal D, Hlobil H, Wassenberg D, Soutschek E: Seroreactivity to and avidity for recombinant antigens in toxoplasmosis. Clin Diagn Lab Immunol 2005, 12:977-982. 
18. Liu Q, Shang L, Jin H, Wei F, Zhu XQ, Gao H: The protective effect of a Toxoplasma gondii SAG1 plasmid DNA vaccine in mice is enhanced with IL-18. Res Vet Sci 2010, 89:93-97.

19. Wu SM, Huang SY, Fu BQ, Liu GY, Chen JX, Chen MX, Yuan ZG, Zhou DH, Weng YB, Zhu XQ, Ye DH: Seroprevalence of Toxoplasma gondii infection in pet dogs in Lanzhou, Northwest China. Parasit Vectors 2011, 4:64.

20. Macri G, Sala M, Linder AM, Pettirossi N, Scarpulla M: Comparison of indirect fluorescent antibody test and modified agglutination test for detecting Toxoplasma gondii immunoglobulin $\mathrm{G}$ antibodies in dog and cat. Parasitol Res 2009, 105:35-40.

21. Marca MC, Ramos JJ, Loste A, Saez T, Sanz MC: Comparison of indirect immunofluorescent antibody test and modified direct agglutination test methods for detection of Toxoplasma gondii antibodies in adult sheep in Spain. Vet Parasitol 1996, 67:99-103.

22. Hosseininejad M, Azizi HR, Hosseini F, Schares G: Development of an indirect ELISA test using a purified tachyzoite surface antigen SAG1 for sero-diagnosis of canine Toxoplasma gondii infection. Vet Parasitol 2009, 164:315-319.

23. Selseleh MM, Keshavarz H, Mohebali M, Shojaee S, Modarressi M, Eshragian M: Production and evaluation of Toxoplasma gondii recombinant surface antigen 1 (SAG1) for serodiagnosis of acute and chronic Toxoplasma infection in human sera. Iranian J Parasitol 2012, 7:1-9.

24. Kawada T: Receiver operating characteristic curve analysis, sensitivity comparison and individual difference. Clin Radiol 2012, 67:940.

25. Li Y, Liu Q, Li S, Wei F, Jin H, Yang M: Seroprevalence of Toxoplasma gondii infection in dogs in Jiangsu Province, eastern China. J Parasitol 2012, 98:878-879.

26. Tsai YJ, Chung WC, Fei AC, Hong CL, Tsai YY, Peng S, Wu YL: Prevalence of Toxoplasma gondii antibodies in stray dogs in Taipei, Taiwan. J Parasitol 2008, 94:1437.

27. Wanha K, Edelhofer R, Gabler-Eduardo C, Prosl H: Prevalence of antibodies against Neospora caninum and Toxoplasma gondii in dogs and foxes in Austria. Vet Parasitol 2005, 128:189-193.

28. Cedillo-Pelaez C, Diaz-Figueroa ID, Jimenez-Seres MI, Sanchez-Hernandez G, Correa D: Frequency of antibodies to Toxoplasma gondii in stray dogs of Oaxaca, Mexico. J Parasitol 2012, 98:871-872.

29. Meireles LR, Galisteo AJ Jr, Pompeu E, Andrade HF Jr: Toxoplasma gondii spreading in an urban area evaluated by seroprevalence in free-living cats and dogs. Trop Med Int Health 2004, 9:876-881.

30. Zhu C, Cui L, Zhang L: Comparison of a commercial ELISA with the modified agglutination test for detection of Toxoplasma gondii antibodies in sera of naturally infected dogs and cats. Iranian J Parasitol 2012, 7:89-95

31. Saraei M, Shojaee S, Esmaeli A, Jahani-Hashemi H, Keshavarz H: Evaluation of confounders in toxoplasmosis indirect fluorescent antibody assay. Iranian J Parasitol 2010, 5:55-62.

32. Holec-Gasior L: Toxoplasma gondii recombinant antigens as tools for serodiagnosis of human toxoplasmosis: current status of studies. Clin Vaccine Immunol 2013, 20:1343-1351.

33. Pietkiewicz H, Hiszczynska-Sawicka E, Kur J, Petersen E, Nielsen HV, Stankiewicz M, Andrzejewska I, Myjak P: Usefulness of Toxoplasma gondii-specific recombinant antigens in serodiagnosis of human toxoplasmosis. J Clin Microbiol 2004, 42:1779-1781.

34. Nigro M, Gutierrez A, Hoffer AM, Clemente M, Kaufer F, Carral L, Martin V, Guarnera EA, Angel SO: Evaluation of Toxoplasma gondii recombinant proteins for the diagnosis of recently acquired toxoplasmosis by an immunoglobulin G analysis. Diagn Microbiol Infect Dis 2003, 47:609-613.

35. Kotresha D, Poonam D, Muhammad Hafiznur Y, Saadatnia G, Nurulhasanah O, Sabariah O, Tan SY, Izzati Zahidah AK, Rahmah N: Recombinant proteins from new constructs of SAG1 and GRA7 sequences and their usefulness to detect acute toxoplasmosis. Trop Biomed 2012, 29:129-137.

36. Neudeck A, Stachelhaus S, Nischik N, Striepen B, Reichmann G, Fischer HG: Expression variance, biochemical and immunological properties of Toxoplasma gondii dense granule protein GRA7. Microbes Infect 2002, 4:581-590.
37. Fischer HG, Stachelhaus S, Sahm M, Meyer HE, Reichmann G: GRA7, an excretory 29 kDa Toxoplasma gondii dense granule antigen released by infected host cells. Mol Biochem Parasitol 1998, 91:251-262.

38. Swets JA: Measuring the accuracy of diagnostic systems. Science 1988 240(4857):1285-1293.

39. Greiner M, Pfeiffer D, Smith RD: Principles and practical application of the receiver operating characteristic analysis for diagnostic tests. Prev Vet Med 2000, 45:23-41.

doi:10.1186/1746-6148-10-158

Cite this article as: Wang et al:: Evaluation of recombinant granule antigens GRA1 and GRA7 for serodiagnosis of Toxoplasma gondii infection in dogs. BMC Veterinary Research 2014 10:158.

\section{Submit your next manuscript to BioMed Central and take full advantage of:}

- Convenient online submission

- Thorough peer review

- No space constraints or color figure charges

- Immediate publication on acceptance

- Inclusion in PubMed, CAS, Scopus and Google Scholar

- Research which is freely available for redistribution

Submit your manuscript at www.biomedcentral.com/submit
C Biomed Central 